\title{
Arm Based Accident Preventive System
}

\author{
Gopal Krishna and Sahadev Roy
}

\begin{abstract}
In this paper, a Controller Area Network (CAN) based communication system is presented for collision or accident avoidance. This system can be used in automobiles to provide more safety. CAN architecture has been proposed and implemented using MCP2515. The major advantage of CAN based smart network over the traditional point to point schemes is increased and more flexibility and expandability new features. Various parameters of the car basically speed, distance from the other cars, obstacles like persons and presences of animals detected and measures accurately. A status signal may send to the car driver if any of the desire parameter goes out of range to avoid accidents. The aim of this project is to avoid collision by detecting obstacles, vehicles using IR or ultrasonic sensors for obstacle avoidance and controlling the vehicle accordingly by using CAN protocol.
\end{abstract}

Keywords

Accident preventive system;

Arm7;

Control Area Network (CAN);

Cyclic Redundancy Check (CRC);

Obstacle detection.

\section{INTRODUCTION}

Accidents occur due to absent mind of driver. An intelligent system is desires to be developed to overcome it [1]. A collision avoidance system is required to place within a car to warn driver like the car is closed to other cars surrounding it and automatic speed is required to reduce while going around a curve, etc. The most of the intelligent car systems used to monitor accident contrl system only [2]. Market price of such automatic systems enable cars are presently huge cost and available in sports cars and other luxury cars only, which are not affordable to everyone [3]. So, a system needs to be developed which can be implemented in car at very low cost. Intelligent collision avoidance systems for automobiles can be possible using the controller Area Network (CAN) protocol [4]. CAN is widely used in a wide range of applications, such as in vehicle communication, automated manufacturing and distributed process control environments [5]. Mostly used in industry and auto mobiles in a Hazardous Environment and is reliable [6].

This paper is arrange as overview of the CAN system is given in section II, hardware design described in section III.

\section{OVERVieW OF CAN Protocol}

Controller area network (CAN) can be used in real time environment due to high speed performance. realtime performance with very low cost [7]. CAN is implemented node to node data transition.

Serial data communication protocol using CAN bus is invented in 1983 by German BOSCH Corporation. Multi-master communication protocol is developed based on serial asynchronous methodology [8]. This protocol is suitable for connecting different electronic modules in industrial applications. CAN supports data frames with sizes only up to 8 bytes which is enough for industrial application due to sensors transmitting small data packets. Up to 64 bits of application data may be transmitted by using TI CAN [9]. CAN is fulfilling the condition of ISO11898:2003 Standard. Its standard 11-bit identifier provides for signalling rates minimum $125 \mathrm{kbps}$ to 1 Mbps. The extended 29-bit identifier was introduced latter. The standard 11-bit identifier field provides 2048 different message identifiers, by using extended 29 - bit identifier provide $2^{29}$ or 537 million identifiers. The over view of CAN frame structure is shown in Fig 1. Data frame of the CAN mainly consist of seven fields, start frame, arbitration field, control field, data field, cyclic redundancy check (CRC) field, acknowledgement (ACK) field and end frame indicator.

\begin{tabular}{|c|c|c|c|c|c|c|}
\hline $\begin{array}{c}\text { Start } \\
\text { Frame }\end{array}$ & $\begin{array}{c}\text { Arbitration } \\
\text { Field }\end{array}$ & $\begin{array}{c}\text { Control } \\
\text { Field }\end{array}$ & $\begin{array}{c}\text { Data } \\
\text { Field }\end{array}$ & $\begin{array}{c}\text { CRC } \\
\text { Field }\end{array}$ & $\begin{array}{c}\text { ACK } \\
\text { Field }\end{array}$ & $\begin{array}{c}\text { End } \\
\text { Frame }\end{array}$ \\
\hline
\end{tabular}

Fig 1. Data frame structure of CAN protocol.

Between the data field and the acknowledgement field there is an error-check field [10]. This field trigger the receiving system's error checking process. If any error detect retransmission request is send to the source node.

A 16 bits cyclic redundancy check (CRC) code is generated for this purpose. Since, CAN use a large 
amount of overhead to make it more secure and reliable 15-bit CRC is used.

Bitwise arbitration process is used in CAN protocol. This arbitration process is non-destructive process which is desired for access any shared resource. The transmission of the identifier field starts the arbitration process [11]. The bus states CAN protocol define a logic bit 0 as a transmitting node monitors and compares the received bit stream with the transmitted bit stream [12]. Depending on the dominant bit, which is received during the transmission, the node arbitration is stops transmission. Actually, transmission process stops by means of lost arbitration process.

\section{A. Reliability}

High error handling capability of CAN bus provide a reliable system. Less chance for failures occurred in any operational interval. If any message transmitting node is identify an error by the strong error checking process, the node effectively aborts transmission and attempts to retransmit repeatedly until its message is received successfully [13]. This functionality lads the CAN bus into hogged stage, if the high priority node is failed. To avoid such failure, receive error counter (REC) and transmit error counter (TEC) are deployed. If such dead loop detected automatically rejected the prior loop for transmission and a failure message send to the operator [14]. Hence, it is required to ensure that no any message node hogs the bus.

One of the widely CAN controller is MCP2515. It has both TEC and REC [15]. Both the counter enhances reliability of the system. The basic states of the CAN controller are error active, error passive or bus off state. The basic operating state of the controller is controlled and monitor by TEC and REC. Error active, error passive or bus off state conditions can be express by Eq. (1), Eq. (2) and Eq. (3) respectively.

$$
\begin{gathered}
\text { Error Active State: }\left\{\begin{array}{c}
T E C \leq 127 \text { and } \\
R E C \leq 127
\end{array}\right. \\
\text { Error Passive State: }\left\{\begin{array}{c}
127<T E C<255 \text { or } \\
127<R E C \\
\text { Bus off State: } 255<T E C
\end{array}\right.
\end{gathered}
$$

When the transmit error counter is greater than 255 bus off state activated and fault indicator status become on. Once the CAN controller has cross the threshold of the bus off state, it must be reset by the host microcontroller in order to be able to continue operation.

\section{HARDWARE DESIGN}

To collision avoidance device is implemented here by using obstacle detection sensors like infra-red (IR) and ultra sonic sensor. For implementation and prototype development we used two nodes which are developed using LPC2148 ARM7 based micro controller and MCP2515 as CAN controller. Block diagrams of the proposed system is shown in Fig. 2. MCP2551 is used as CAN transrecever. The Can protocol is implemented using SPI lines of ARM7.

The Node I are used for obstacle detection and second Node II consider DC motor for vehicle prototypes. For any obstacle is appear in front of Node I, a stop signal will be produced in Node II by CAN protocol.

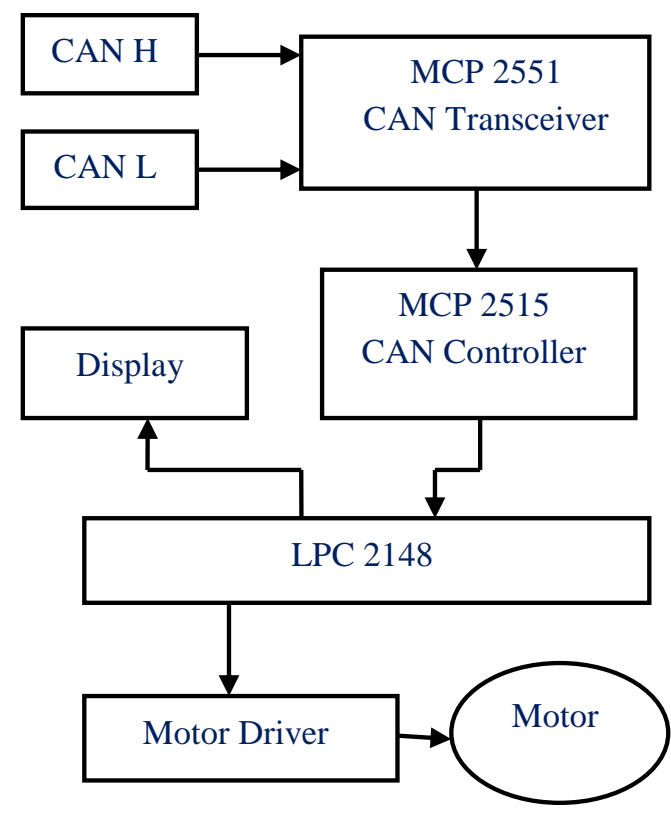

Fig 2. Block diagram of the proposed CAN base system.

\section{A. Can Bus Electrical Configuration}

CAN transmission medium are formed by lowlevel transmission line (CANL) and high-level transmission line $(\mathrm{CANH})$ and connected to $\mathrm{CANH}$ (pin 6) and CANL (pin 7) pins of MCP2551 CAN transceiver. The difference between $\mathrm{CANH}$ and CANL with respect to ground is called here $\mathrm{V}$ diff. The host processor take actions when it received messages and action taken accordingly. The output

Gopal Krishna and Sahadev Roy, "Arm Based Accident Preventive System," International Journal of Advanced Engineering and Management, Vol. 2, No. 7, pp. 176-179, 2017.

DOI: https://doi.org/10.24999/IJOAEM/02070040 
devices are consider motor driver. According to the output of Node I, a warning signals generate and the driver take appropriate action.

The CAN controller expected that the transmitted by CAN transceiver MCP2551. CAN transceiver MCP2551 received the data from the sensor and transforms it to It do the same operation to the CAN bus. The data which are transmitted to the CAN controller well synchronized. The first and fourth pins of the MCP2551 is TxD and RxD repetitively (Fig. 3). This two pins are connected to the CAN controller where CANH and CANL is connected to the CAN bus.

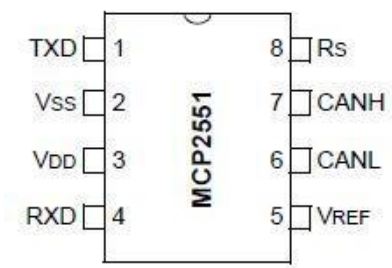

Fig 3. Pin diagram of $M C P 2551$.

Algorithm for Transmitter and Receiver Algorithm for transmitter side which consists sensors,ARM7 microcontroller and CAN (MCP2515) is as follows:

Initialize SPI (Serial Peripheral Interface).

Initialize CAN (MCP2515).

Impulse signal generation for ultrasonic sensor.

Distance measurement from other car and display on LCD.

Send acknowledgment to the transmitter.

Receive distance data from CAN of transmitter and if distance is less then display warning signal on LCD.

If is received then display Car will be stopped.

\section{CONCLUSION}

The proposed device is best suitable for avoiding collisions in automobiles. Flexibility Real-time performance and reliability, all these performance make CAN in car manufacturing industry. Using CAN protocol, internodes communication and node to other sensors and actuators module communication is possible. The proposed methodology ensures low power, low cost and high performance real time operation and control of automobile accident control mechanism.

\section{REFERENCES}

[1] Dinges, D. F. (1995). An overview of sleepiness and accidents. Journal of sleep research, 4(s2), 414.

[2] Jones, W. D. (2001). Keeping cars from crashing. IEEE spectrum, 38(9), 40-45.

[3] Sheller, M., \& Urry, J. (2000). The city and the car. International journal of urban and regional research, 24(4), 737-757.

[4] Wang, A. P., Chen, J. C., \& Hsu, P. L. (2004, March). Intelligent CAN-based automotive collision avoidance warning system. In Networking, Sensing and Control, 2004 IEEE International Conference on (Vol. 1, pp. 146151). IEEE.

[5] Gungor, V. C., Sahin, D., Kocak, T., Ergut, S., Buccella, C., Cecati, C., \& Hancke, G. P. (2013). A survey on smart grid potential applications and communication requirements. IEEE Transactions on Industrial Informatics, 9(1), 28-42.

[6] Srivastava, R., Kumar, N., Barnwal, A., \& Singh, S. (2017). Grid Interactive Solar Powered Automated Bottling Plant Using Microcontroller. International Journal of Advanced Engineering and Management, 2(1), 9-14.

[7] Leen, G., \& Heffernan, D. (2002). Expanding automotive electronic systems. Computer, 35(1), 88-93.

[8] Craessaerts, G., Maertens, K., \& De Baerdemaeker, J. D. (2005). A Windows-based design environment for combine automation via CANbus. Computers and electronics in agriculture, 49(2), 233-245.

[9] http://www.ti.com/lit/an/sloa101b/sloa101b.pdf.

[10] Bruckert, W. F., Bissett, T. D., Mazur, D., \& Munzer, J. (1993). U.S. Patent No. 5,255,367. Washington, DC: U.S. Patent and Trademark Office.

[11] Saha, R., Nandi, V., Roy, S., \& Bhunia, C. T. (2016). Design and verifications of efficient arbiter of SoC's on-chip bus. In Proceeding of IEEE 3rd International Conference on Electronics and Communication Systems (pp. 989-92).

[12] Farsi, M., Ratcliff, K., \& Barbosa, M. (1999). An overview of controller area network. Computing \& Control Engineering Journal, 10(3), 113-120.

[13] Liskov, B., Curtis, D., Johnson, P., \& Scheifer, R. (1987). Implementation of argus. ACM SIGOPS

Gopal Krishna and Sahadev Roy, "Arm Based Accident Preventive System," International Journal of Advanced Engineering and Management, Vol. 2, No. 7, pp. 176-179, 2017.

DOI: https://doi.org/10.24999/IJOAEM/02070040 
Operating Systems Review, 21(5), 111-122.

[14] Thomson, T. W. (1996). U.S. Patent No. 5,574,848. Washington, DC: U.S. Patent and Trademark Office.

[15] Joshi, K., \& Gohil, V. (2014). ARM 7 based controller area network for accident avoidance in automobiles. arXiv preprint arXiv:1403.3156.

\section{Author Details}

Gopal Krishna

Dept. of Electronics and Communication Engineering, NIT Arunachal Pradesh, Yupia, India

Email: krishna100491@gmail.com

Sahadev Roy

Dept. of Electronics and Communication Engineering,

NIT Arunachal Pradesh, Yupia, India

Email: sdr.ece@nitap.in

Gopal Krishna and Sahadev Roy, "Arm Based Accident Preventive System," International Journal of Advanced Engineering and Management, Vol. 2, No. 7, pp. 176-179, 2017. 